$\ddot{1}$ STFM SOCIETY OF TEACHERS OF From the Society of Teachers FAMILY MEDICINE of Family Medicine

Ann Fam Med 2018;16:278. https://doi.org/10.1370/afm.2251.

\section{STFM AND ABFM PILOT PRECEPTING PERFORMANCE IMPROVEMENT PROGRAM}

On April 2, the Society of Teachers of Family Medicine and the American Board of Family Medicine (ABFM) kicked off a pilot program that offers Performance Improvement continuing certification credit (previously MOC Part IV) to ABFM diplomates who provide personal instruction, training, and supervision to a medical student or resident and who participate in a teaching improvement activity.

The Precepting Performance Improvement Program allows academic units (Sponsors), who were selected through an application process, to develop and oversee the completion of performance improvement (PI) projects that meet the ABFM Family Medicine Certification requirements. Sponsors develop and oversee PI projects for teaching physicians without having to submit an application for each activity for ABFM review.

ABFM is piloting the program through December 2018. The program will be modified as needed based on input from the pilot sites, then made available to all academic units/preceptors.

Sponsors selected to participate in the pilot are:

- 55th Medical Group, Offutt AFB, NE

- Albany Medical College, Albany, NY

- Albert Einstein College of Medicine, Montefiore Medical Center, Bronx, NY

- Baylor College of Medicine, Houston, TX

- Cook County Health and Hospital System, Chicago, IL

- DHR Family Medicine Residency, McAllen, TX

- Ehrling Bergquist Clinic Family Medicine Residency, Bellevue, NE

- Ellis Medicine Family Medicine Residency, Schenectady, NY

- Emory Family Medicine Student Programs, Atlanta, GA

- Florida Atlantic University, Boca Raton, FL

- Florida International University, Miami, FL

- HealthAlliance Fitchburg Family Practice, Fitchburg, MA

- Louisiana State University HSC, Lake Charles, LA

- New York Medical College at Saint Joseph's Medical Center Family Medicine Residency, Yonkers, NY

- Penn State College of Medicine, Hershey, PA

- Penn State College of Medicine, State College, PA
- Quinnipiac University, Hamden, CT

- Saint Francis Hospital Residency, Wilmington, DE

- Samaritan Family Medicine Residency, Corvallis, OR

- Temple University, Philadelphia, PA

- Texas Tech Family Practice Residency, Amarillo, TX

- Texas Tech Family Practice Residency, El Paso, TX

- The University of Kansas Medical Center, Kansas City, KS

- Trident/MUSC Family Medicine Residency, Charleston, SC

- UCSF Natividad Family Medicine Residency, Salinas, CA

- UHS Wilson Family Medicine Residency, Johnson City, NY

- University of California, Davis, CA

- University of California, Riverside, CA

- University of Kentucky, Lexington, KY

- University of Minnesota, Twin Cities campus, MN

- University of Mississippi Medical Center, Jackson, MS

- University of Missouri, Columbia, MO

- University of North Carolina, Chapel Hill, NC

- University of North Dakota, Grand Forks, ND

- University of Oklahoma-Tulsa, Tulsa, OK

- University of South Carolina, Greenville, SC

- University of Utah, Salt Lake City, Utah

- Virginia Commonwealth University, Richmond, VA

- West Virginia University, Morgantown, WV

- Western Michigan University, Kalamazoo, MI

- Zucker School of Medicine at Hofstra/Northwell, Hempstead, NY

\section{Preceptor Expansion Initiative}

The STFM/ABFM Pilot Program is part of a wider initiative, being led by STFM, to address the shortage of clinical training sites for students. Five interprofessional, interdisciplinary teams are working on the following tactics:

Tactic 1: Work with CMS to revise student documentation guidelines

Tactic 2: Integrate interprofessional/interdisciplinary education into ambulatory primary care settings

Tactic 3: Develop standardized onboarding process for students and preceptors and integrate students into the work of ambulatory primary care settings in useful and authentic ways

Tactic 4: Develop educational collaboratives across departments, specialties, professions, and institutions to improve administrative efficiencies

Tactic 5: Promote productivity incentive plans that include teaching and develop a culture of teaching in clinical settings

Learn more and follow the progress of the initiative at http://www.stfm.org/Resources/Resourcesfor MedicalSchools/PreceptingExpansionInitiative. 